\title{
Supporting Novice Engineers in Idea Generation using Design Heuristics
}

\section{Laura R. Murphy, University of Michigan}

Laura is a senior in Mechanical Engineering at the University of Michigan, Ann Arbor. She is passionate about understanding how design can impact the human condition. Her research surrounds front-end design and how every student can engage with engineering in their own way. She is the co-founder and CEO of Adapt Design, a disability design company creating beautiful products that facilitate emotional and physical independence for people with disabilities. Engineering provides a technical background to demonstrate the social impact possible through design.

\section{Dr. Shanna R. Daly, University of Michigan}

Shanna Daly is an Assistant Professor in Mechanical Engineering at the University of Michigan. She has a B.E. in Chemical Engineering from the University of Dayton (2003) and a Ph.D. in Engineering Education from Purdue University (2008). Her research focuses on strategies for design innovations through divergent and convergent thinking as well as through deep needs and community assessments using design ethnography, and translating those strategies to design tools and education. She teaches design and entrepreneurship courses at the undergraduate and graduate levels, focusing on front-end design processes.

\section{Dr. Seda McKilligan, Iowa State University}

Dr. McKilligan is an Associate Professor of Industrial Design. She teaches design studios and lecture courses on developing creativity and research skills. Her current research focuses on identifying impacts of different factors on ideation of designers and engineers, developing instructional materials for design ideation, and foundations of innovation. She often conducts workshops on design thinking to a diverse range of groups including student and professional engineers and faculty member from different universities. She received her PhD degree in Design Science in 2010 from University of Michigan. She is also a faculty in Human Computer Interaction Graduate Program and the ISU Site Co-Director for Center for e-Design.

\section{Dr. Colleen M. Seifert, University of Michigan}

Colleen M. Seifert is an Arthur F. Thurnau Professor in the Department of Psychology at the University of Michigan. She received her Ph.D. in Cognitive Science and psychology at Yale University. She was an ASEE postdoctoral fellow at the University of California - San Diego and the Navy Personnel Research Development Center. Her research interests center on learning, memory, and creativity. 


\title{
Supporting Novice Engineers in Idea Generation using Design Heuristics
}

\author{
Laura Murphy \\ University of Michigan, Ann Arbor, MI, USA \\ Shanna R. Daly \\ University of Michigan, Ann Arbor, MI, USA \\ Seda Yilmaz \\ Iowa State University, Ames, IA, USA \\ Colleen M. Seifert \\ University of Michigan, Ann Arbor, MI, USA
}

\begin{abstract}
How can beginning engineers learn to generate a variety of candidate concepts to consider? Because they likely have little experience with idea generation, training in specific techniques may be especially useful. Design Heuristics are an evidence-based tool developed to help engineers expand diversity of ideas considered during the front-end phase of design. Brainstorming is a common ideation technique in both engineering education and practice. Our research sought to compare the characteristics of ideas generated by incoming engineering freshmen using both techniques. The analyses of this study involve qualitative patterns in a subset of data collected from 94 incoming engineering freshmen. We explored diversity, fixation, and the scope of the concepts generated. Ideas were coded based on a variety tree coding scheme, students' selfperceptions, and system vs. component scope. We found that concepts generated using Design Heuristics were more likely to focus on the methods for achieving functions and on specific components, while Brainstormed ideas tended towards more holistic systems. The results suggest that alternative tools for idea generation may serve to focus attention on different qualities of design, and that multiple tools may be important for design in engineering education.
\end{abstract}

\section{Introduction}

Engineering design has been described as a problem-solving process that "searches through a hypothetical space of many possible ideas."'[1, 2] A design process can be viewed as having a frontend and a back-end, where the front-end design is a "fuzzy" process full of ambiguity[3] that involves a series of divergent and convergent thinking episodes in order to come to a conceptual design[4]. The front-end phase includes defining problems, conducting research and design ethnography, identifying design requirements, idea generation, and initial prototyping[3, 4, 5]. Engineering curricula often focus on the "back-end" of design, where processes focus on developing, building, and testing concepts[6], potentially because these processes appear deterministic (such as optimizations). However, many crucial design decisions are made in the front-end phase[7], and skills in front-end design are necessary to successful design. This study focuses on one important stage in the front-end design: idea generation; in particular, what are strategies and tools that can be used to support front-end success in design? 
One of the challenges for both engineering students and practicing engineers is to develop innovative solutions to problems because innovation is necessary to solve the world's most pressing issues.[8] As increasing technology allows engineers to learn more about existing and emerging problems, it also provides opportunities to develop solutions like never before.[9] Consequently, engineers must be successful in idea generation $[8,10]$ by fully exploring solution spaces through the generation of multiple and diverse ideas[11]. Considering a wide spectrum of ideas at the front end of the design process is important in order to consider strong aspects of concepts to develop while filtering out other aspects. Generating diverse ideas to consider requires divergent thinking, $[4,12]$ while engineering methods focus on converging on a single solution. While experienced designers appear to use strategies in their idea generation process[13, 14], novice designers may have fewer approaches to help them consider a variety of concepts.

One way to assist novice engineers in idea generation is through idea generation tools. A common tool is Brainstorming, the technique of generating ideas without evaluating them, encouraging wild and exaggerated ideas, valuing quantity over quality, and considering every idea to be of equal worth[15]. Brainstorming is the most popular tool used across all disciplines as a way to generate ideas[16]. One challenge with Brainstorming, however, is that it fails to provide students direction or inspiration beyond their initial ideas. Another design tool with increasing use in engineering contexts is Design Heuristics, which are a set of 77 strategies to incorporate into product design idea generation[17]. The Design Heuristics ideation tool is derived from evidence of strategy use by engineers, and has been studied across contexts including undergraduate and graduate engineering, industrial designers, high school engineering education, and professional engineering and design practices[13]. For this study of idea generation in beginning engineers, we compare these two ideation methods to examine qualitative differences in outcomes.

\section{Background}

Research indicates the importance of considering multiple concepts in the early stages of design.[3] However, many engineering students do not dedicate adequate time to the idea generation process. This leads to limited consideration of alternative solutions, and can potentially decrease the chance of innovative outcomes. Further, designers often become fixated on their initial ideas, and find it difficult to think of very different concepts[18, 19]. Engineering students have been shown to struggle with fixation to a greater extent when compared to industrial design students[18]. Engineering students in particular may benefit from the use of idea generation tools to help them push beyond initial ideas to solutions outside the obvious[20].

One ubiquitous approach to idea generation across disciplines is Brainstorming, a technique originally proposed for groups to help postpone judgment of ideas, encourage wild ideas, aim for quantity over quality, and value every idea[15]. As originally proposed by Osborn), Brainstorming was developed as a technique to use with groups[15]. In practice, "brainstorming” refers to any method of idea generation where groups or individuals are instructed to generate as many different ideas as possible[21]. Engineering instructors, in many cases, encourage their students to generate ideas using "brainstorming," but may not provide students with specific instruction on how to execute it (following Osborn). Instead, the term may be used to suggest a "natural" approach to thinking of ideas, pursuing whatever comes to mind in the moment. The expectation is often that ideas should arise without using any cognitive strategy, and without any instructions on how to 
ideate successfully. This lack of instruction often prevents novice engineers from considering possible strategies as they create and develop concept ideas[14]. As a result, students are less likely to recognize the large space of potential designs possible, and may not seek out tools to help them identify varied candidate designs. The instructions in Brainstorming [15] push designers to continue creating more solutions; however, they do not guide or direct towards potential solutions. This lack of direction also causes students to rely on their initial ideas[34].

Another idea generation approach is Design Heuristics, an evidence-based concept generation tool developed to help designers think systematically and intentionally about variety in idea generation[20, 22]. Collected from protocols of engineers talking aloud while creating designs, analyses of award winning products, and a case study of a long-term design project, Design Heuristics capture the cognitive "rules of thumb" used by designers to intentionally vary their set of candidate designs[23]. These strategies appear to be ones that expert designers employ automatically, without consciously deciding to do so[24]. The heuristics were individually extracted across multiple concepts from multiple designers to reflect a useful level of abstraction in describing how to alter design characteristics to create new ones[25]. The resulting set of Design Heuristics capture 77 different strategies, each of which can be applied independently or in to create new designs[26].

The set of Design Heuristics is packaged as an instructional tool for use in idea generation. A set of 77 cards includes prompts, such as "Add Motion," as shown in Figure 1. Each prompt is a different strategy to help the designer generate a novel idea. One side of each card has a graphic demonstrating the strategy along with a written description (Figure 1). The other side includes two examples of the heuristic as employed in two existing products (Figure 1). To illustrate that each heuristic may be applied to every design problem, one of the illustrated products is always a type of chair. The other product on each card varies, demonstrating that these strategies apply across a wide range of applications and industries. While initial use is supported by the cards, it is possible that novices will eventually incorporate the heuristics they often use into their everyday design practice as they develop design expertise[11].

There are many ways that a single Design Heuristic can be applied during idea generation. Past studies have documented their use by means of a single application of one heuristic card to generate a new design, repeated use of one card to generate multiple designs, and combining multiple cards to generate an idea[20]. In addition, students have successfully transformed an existing design into a new one by applying a heuristic[27]. Design Heuristics have also been successful when used in conjunction with other idea generation strategies, such as Morphological Analysis[17].

Research on Design Heuristics has shown that engineering students at varying levels of training can learn to use the Design Heuristics cards within a short instructional session, and then go on to successfully create their own novel and diverse concepts[20]. One study of 48 first-year engineering students given different subsets of 12 Design Heuristics used Design Heuristics in over half of their created concepts for a portable solar oven[28]. Further, the concepts resulting from the application of Design Heuristics were rated by blind coders as more creative designs. Studies with more advanced engineering students showed that design teams made use of their concepts including Design Heuristics in senior capstone projects across various design problems[29]. Even non-engineering students have been shown to be able to apply Design 
Heuristics and produce more creative concepts[22]. Other studies suggest that Design Heuristics can be successfully used by entering engineering students, and found several methods including brainstorming facilitate positive outcomes in idea generation[17].

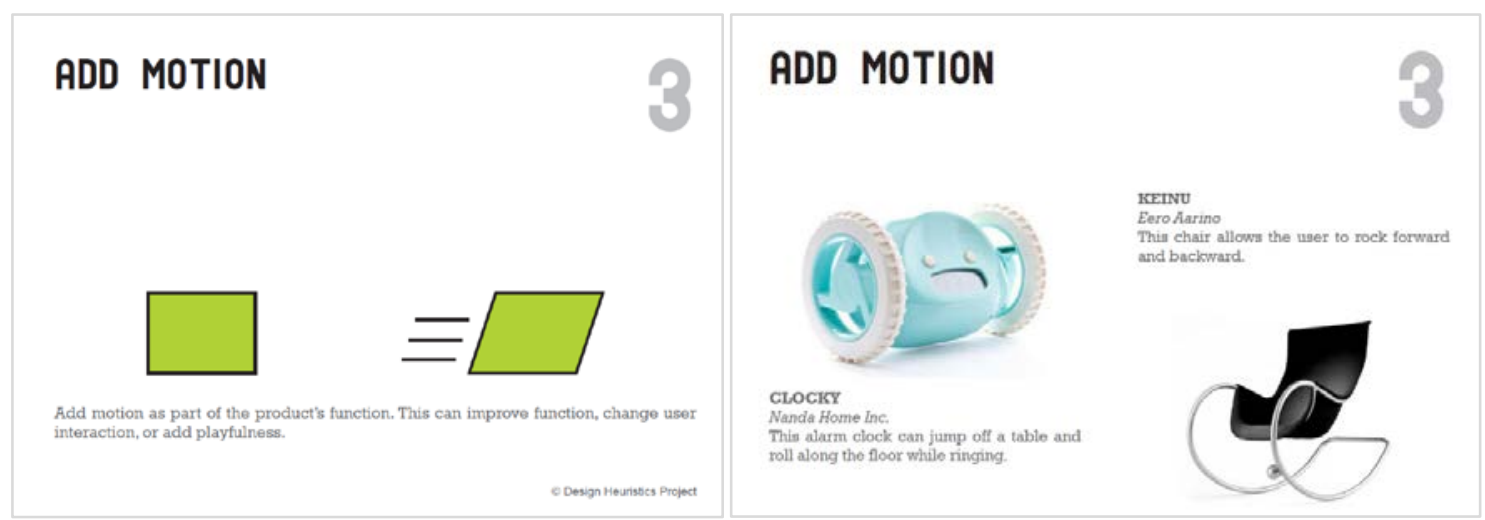

Figure 1: An example of a Design Heuristics card, "Add Motion.” One side of the card shows a graphic image and written description of the strategy; the other side shows two award-winning products that employ the strategy.

\section{Method}

In order to explore how Brainstorming and Design Heuristics might be leveraged in novice designer idea generation, our study was guided by the following research question:

How do the types of ideas generated differ between Brainstorming and Design Heuristics?

To answer this question, participants in a design workshop for entering engineering students were asked to use both ideation methods to generate solutions to presented design problems within a single 75 minute session.

\section{Participants}

The participants were 94 students who were incoming engineering freshmen at a Midwestern American university. These first-year students signed up for a free one-day workshop on design, and this study was one of multiple activities in the workshop. The students had not yet attended any engineering classes at the university. The group was $73 \%$ male, $27 \%$ female.

\section{Procedure}

Data were collected during a two-day immersive design program for incoming engineering freshmen. The sessions were led by advanced design students following a scripted presentation.

First, the students were instructed about idea generation processes. Then, participants were introduced to Brainstorming, including a definition of the technique and a short practice activity using it. Students drew a simple sketch and wrote a short description of the concept's functions, including labels. The students were asked to generate 5 solutions using Brainstorming with following design problem: Design a device to prepare a burrito. They were given 25 minutes to generate concepts, and the facilitator indicated 5-minute time intervals. 
Participants were then instructed on how to use Design Heuristics in a ten-minute instructional session. First, the heuristic cards were introduced, noting the definition, graphic image, and two product examples of its use (see Figure 1). A short practice problem followed where students applied one card to a presented problem. Next, each participant was given the same set of 7 Design Heuristic cards. Due to the time limit of the session, only a subset of cards was provided. This single set of seven cards was chosen at random from the deck of 77 Design Heuristics and included: Scale up or down, Use multiple components for one function, Adjust function through movement, Bend, Reconfigure, Allow user to customize, and Change surface properties. We chose to give a single randomized set to all participants in order to explore variations in the resulting design concepts across participants.

Next, the participants were asked to generate 5 conceptual solutions using Design Heuristics for the same design problem in another 25-minute session (with 5 minute intervals). The two methods were used in the same order - brainstorming then Design Heuristics - because the educational workshop necessitated equivalent learning benefits to all participants. Further, based on prior experience with idea generation by engineering students in design classes, we expected that participants would think of their own solutions first when reading the problem. Previous studies of idea generation have documented the tendency of designers to become "fixated" on initial ideas $[17,19,34]$. So, providing the opportunity to express the initial ideas that "come to mind" might allow students to record them and then move on to consider other concepts.

As a last step in the study, each participant was asked to group their concepts into categories of similar designs. The intent was for the individuals to assess the commonalities emerging from their concepts over their entire set. A survey question asked students:

Consider your set of concepts again. Some concepts may seem related, while others are one of a kind. How many different KINDS of concepts are in your set? Write down each group of similar concepts in your set. Add a label to describe why you put them together. Make sure every concept is listed either in a group or by itself.

The complete session lasted 75 minutes.

\section{Data Analysis}

All of the students completed the exercise within the time frame of the session. We compared the set of 5 possible Brainstorming concepts to the set of 5 possible Design Heuristics concepts. Quantity of ideas has been shown to be a compounding factor in measures of diversity[17]. So, students whose concept counts from the two sessions differed by more than one concept were omitted from the comparison. For example, if a student generated 3 ideas during Brainstorming and 5 during Design Heuristic use, this student's data was eliminated from this analysis. Eighteen datasets were then selected at random from participant datasets for qualitative analysis. Each individual's dataset was assessed as a whole, with an average of 9.6 concepts each. 


\section{Participant Similarity Analysis}

In the final step of the study, each student placed their concepts into groups of similar concepts. The number of categories created by each participant ranged from 2-6, with an average of 4.1. An example of the self-identified categories can be found in Table 1.

An analysis of concept diversity was conducted by counting how many of each student's categories contained an idea generated during Brainstorming compared to how many categories contained concepts generated by Design Heuristics. In this example, there were 3 categories that contain Brainstorming ideas (concepts numbers 1-5), and there were 4 categories that contain Design Heuristics ideas (concept numbers 6-10). This indicated that for Student A, according to their selfperceptions, they generated a somewhat more diverse set of ideas when using Design Heuristics compared with Brainstorming. Further analysis is required to understand the implications this analysis has in larger contexts.Table 1: Example of how one student placed their 10 concepts into 4 categories.

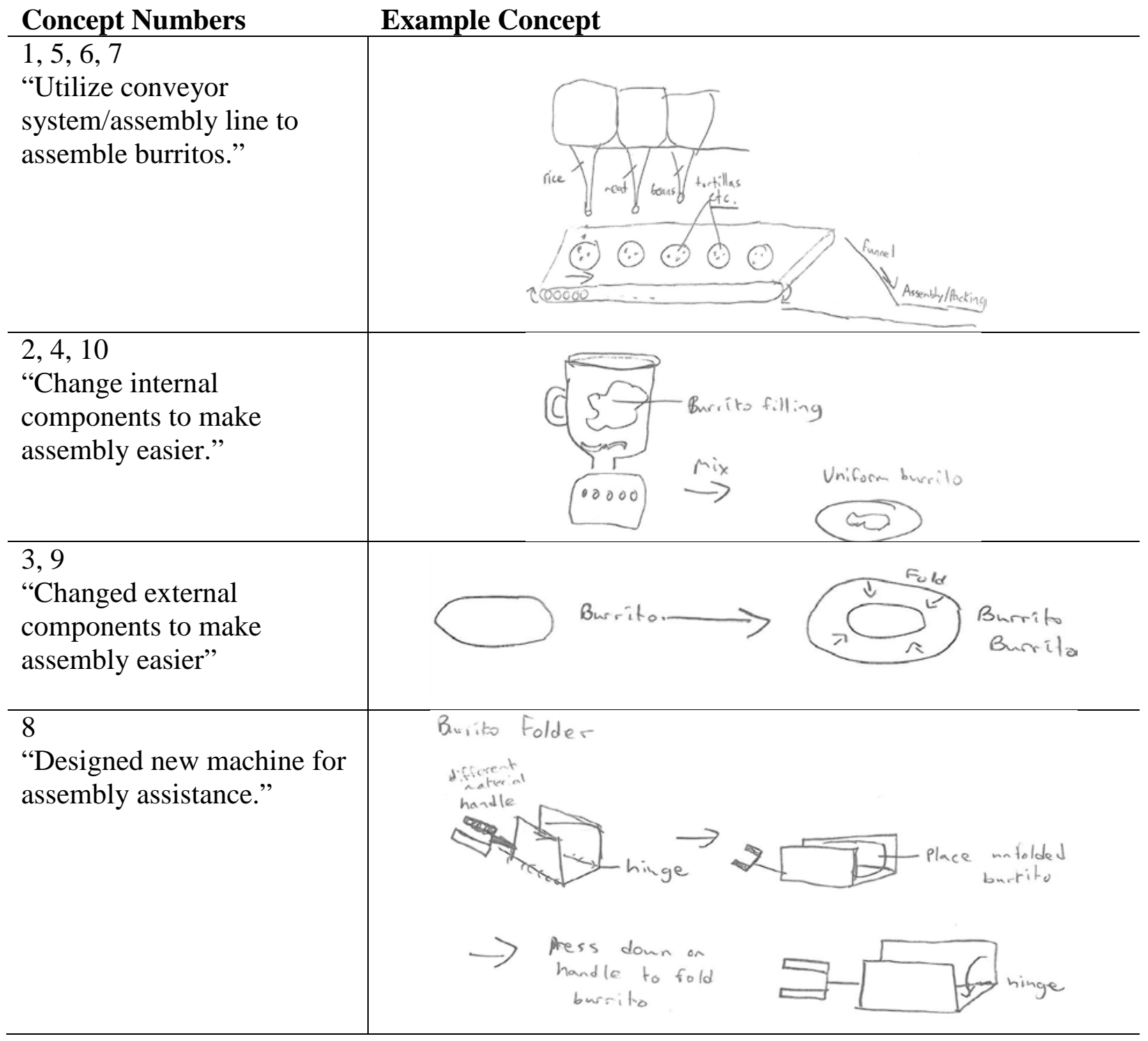


Variety Tree Analysis

In order to understand what types of ideas were generated with Brainstorming vs. Design Heuristics, our analysis attempted to identify the prominent characteristics of the concept sets. In this analysis, we focused on the diversity or variety across a set of ideas to describe how different they are from one another.[30] The data was coded by an advanced undergraduate in Mechanical Engineering.

First, the design problem was broken into 5 main categories based on the most commonly cited characteristics. These 5 main categories describe the functional steps necessary to complete the presented design problem. Some ideas did not fall under any of these categories, and this "Other" category includes concepts where the problem was "reframed;" for example, although the presented problem was design a device to prepare a burrito, a few students stated that they did not need such a device.

Second, we assessed diversity through the creation of a variety tree.[30] This method attempts to characterize the major similarities among designs as distinct categories. A first pass through the total of 164 concepts contained in the 18 datasets identified key functional components and characteristics of the designs. The characteristics that appeared across concepts informed a division of the design concepts into the variety tree shown in Figure 2. For example, 9 concepts described a version of "user inputs information to make the burrito." Additionally, 7 other concepts specified "user input via levers/dials/buttons." This indicated that many concepts involved "user input" more generally, and this category was incorporated in the variety tree.

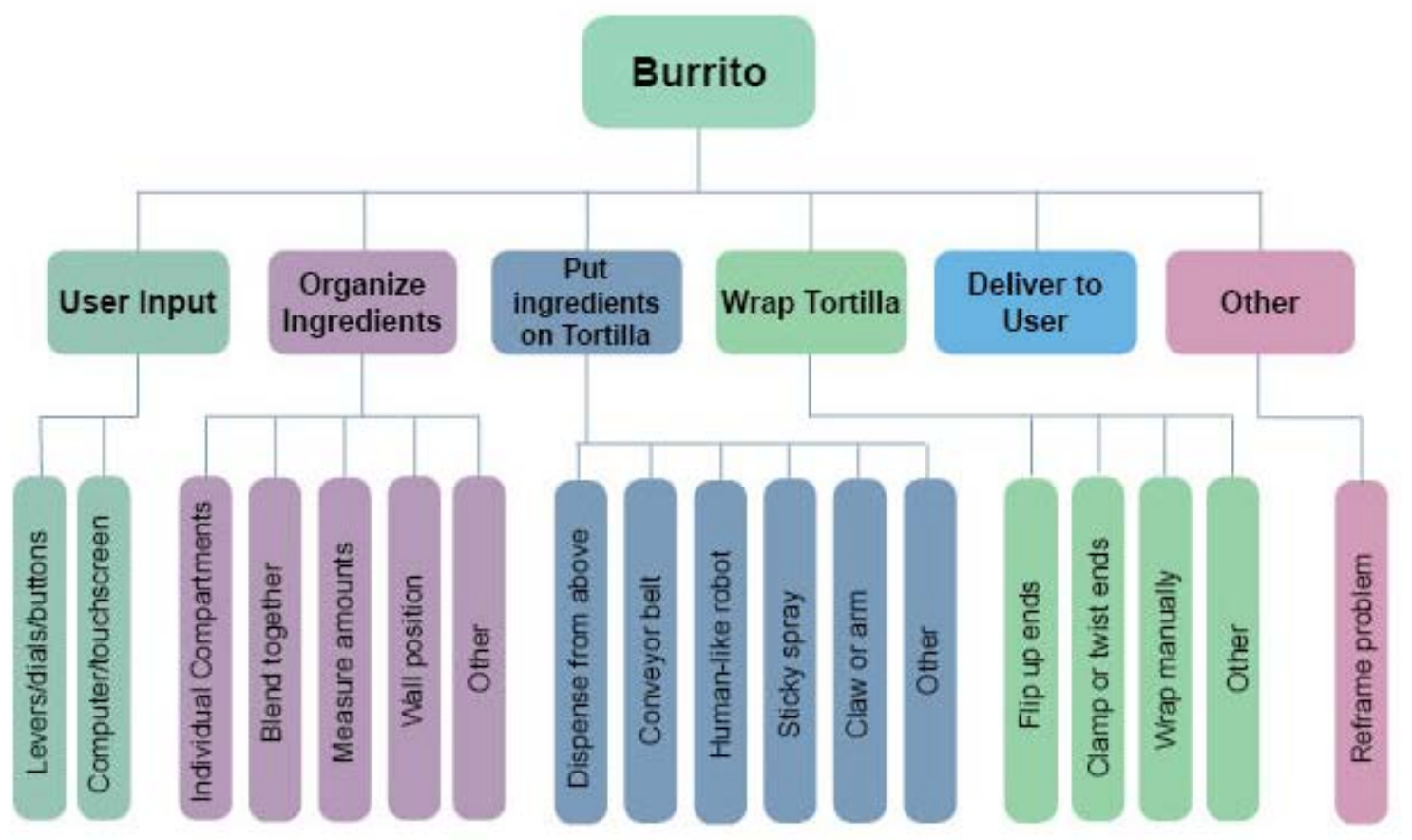

Figure 2: Variety tree for the design problem, "Design a device to prepare a burrito.” The first level includes main functional steps, and the second level includes specific methods to achieve those functions. 
The main categories were then divided into subcategories to describe how concepts achieve each function. The specific methods listed were ones appearing in several concepts. For example, $41 \%$ of the concepts included organizing ingredients by putting them into individual compartments, a subcategory called, "Organize Ingredients." Specified methods that appeared in a single concept were coded into 'other' categories. For "Deliver to User," none of the students specified how they might complete that function, so no subcategories are listed. This coding process was conducted for each of the 164 concepts in the subset analysis.

\section{Results}

To illustrate the variation in the concepts created by students, we present an example of one student's concept set (see Table 2). Concepts 1-5 were generated using Brainstorming, and Concepts 6-10 were generated using Design Heuristics. Each drawing has a corresponding description written by the student. This student's concept set was particularly interesting because of the visual distinction between the Brainstorming concepts and the Design Heuristics concepts. While the Brainstorming concepts sequentially build off each other and are visually represented in the same way, there are few, if any, similarities between each Design Heuristics concept. This indicated that there is a difference between the types of ideas generated, something that we were later able to begin quantifying.

Table 2: Example concepts from a single student, "Student A", including a labelled drawing and a text description for each concept.

Concept Sketch

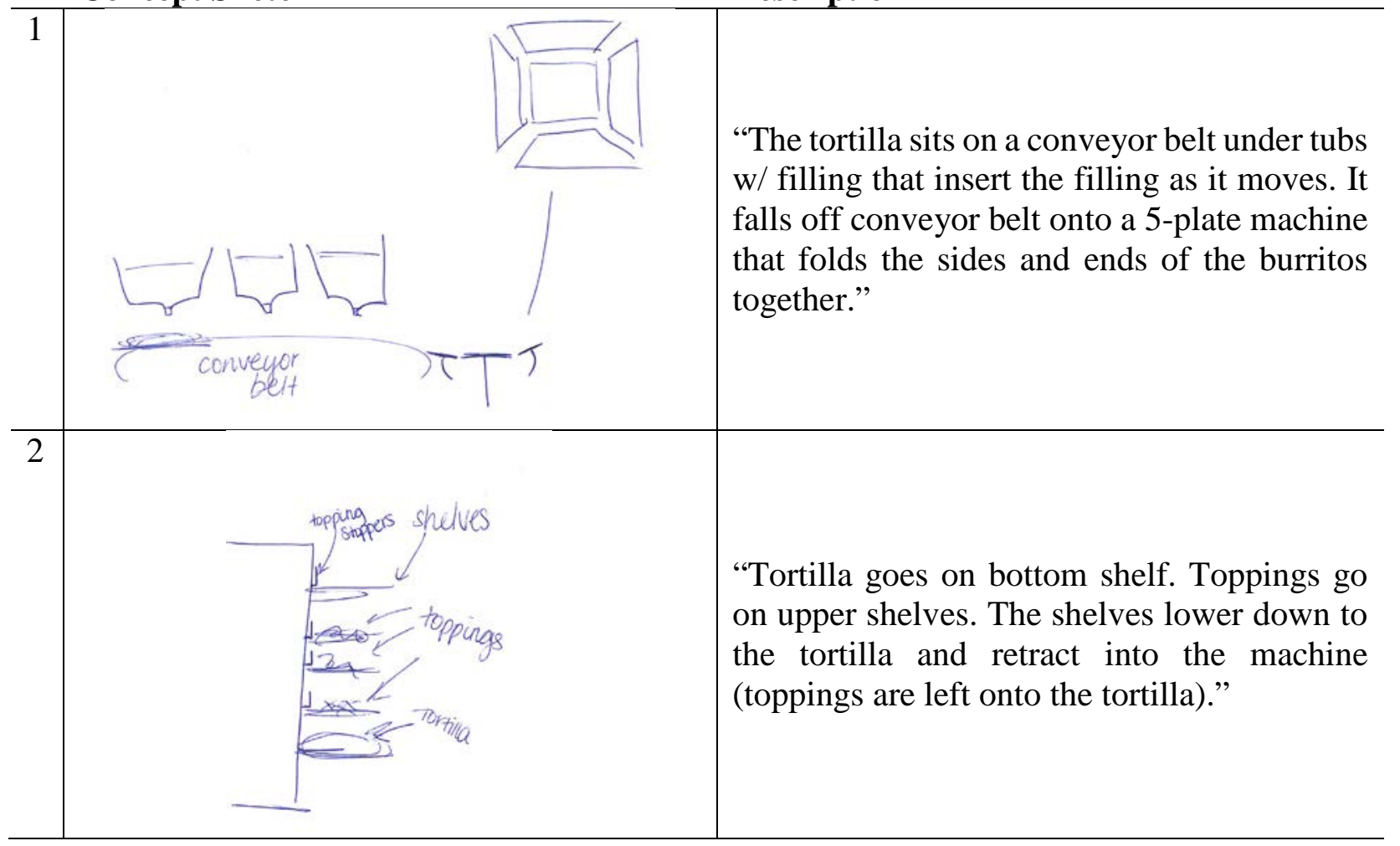




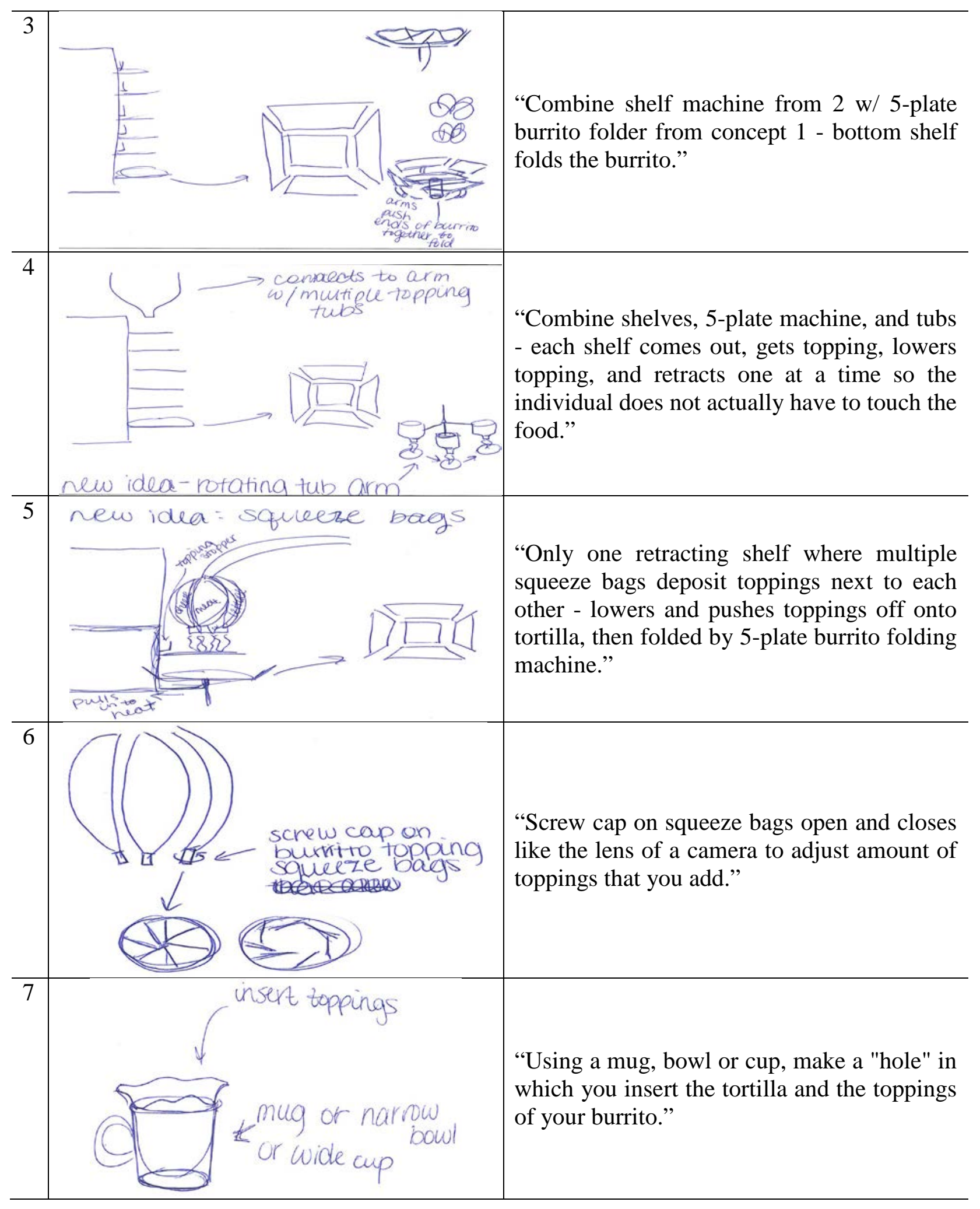




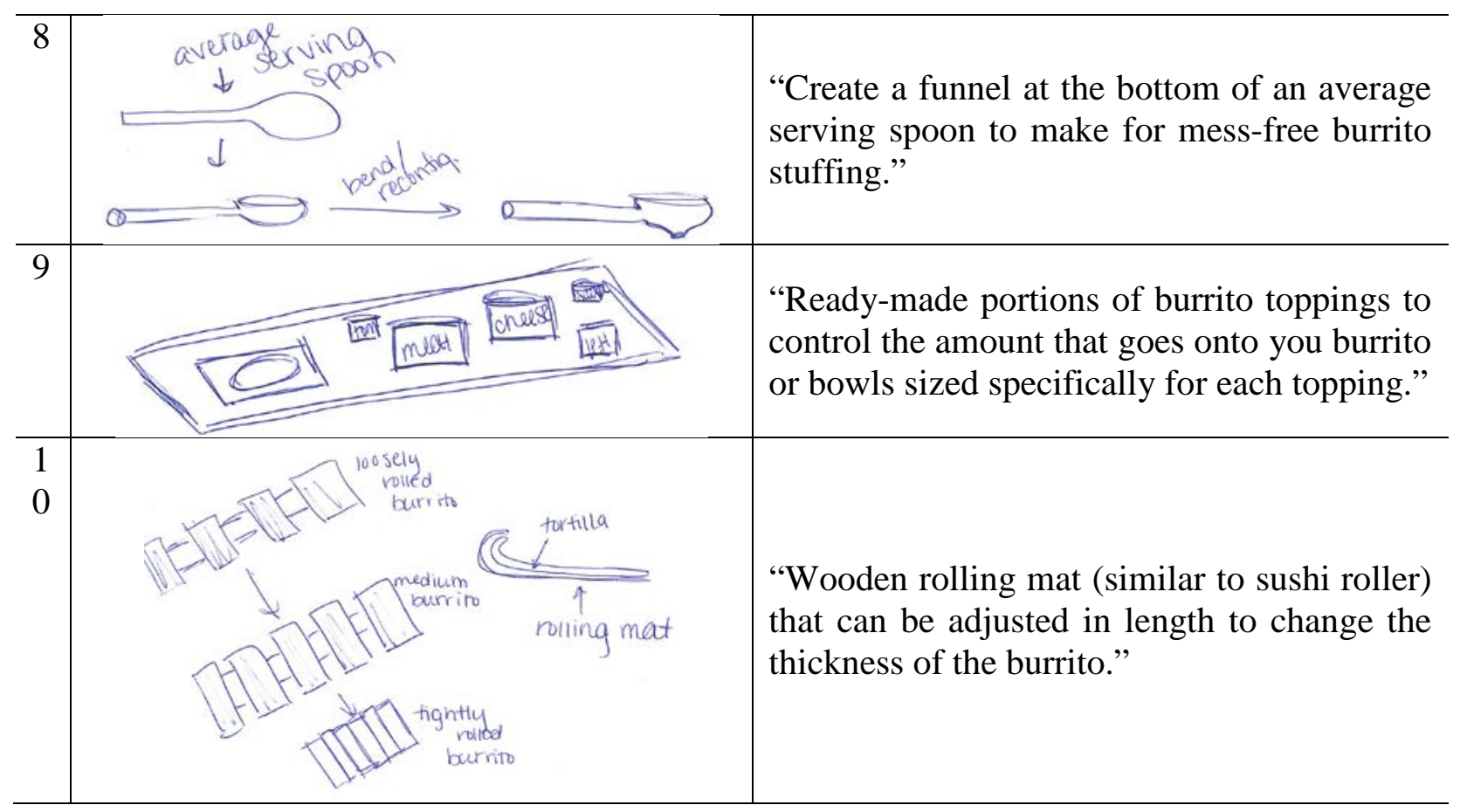

Each of the 164 concepts was coded based on the variety tree; specifically, each was coded for focus on the 5 main process steps and on functional subcategories. For example, Figure 3 shows one of the concepts generated by a different student. This idea has containers of ingredients that dispense onto tortillas on a conveyor belt, and the tortilla is funneled into a packing area where a human assembles it.

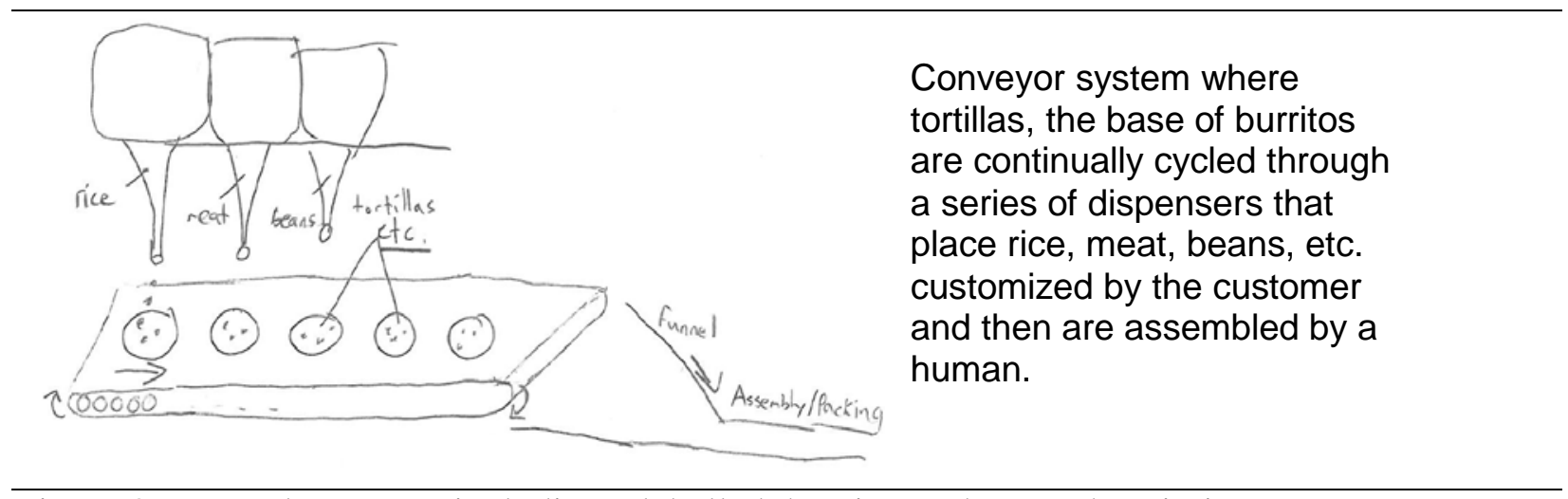

Figure 3: Example concept including a labelled drawing and a text description.

This concept was coded as shown in Figure 4:

- User Input: the concept addresses user input by allowing the customer to customize, but does not specify how exactly this would be accomplished.

- Organize Ingredients: ingredients are stored in individual containers as indicated by the 'rice,' 'beans,' and 'meat,' labels.

- Put Ingredients on Tortilla: ingredients are dispensed from above onto a tortilla, which is on a conveyor belt. 
- Wrap Tortilla: assembly of the tortilla is done by a human as indicated by the written description, hence the concept is coded for 'wrap manually.'

(Deliver to User and Other are not addressed and is therefore not coded.)

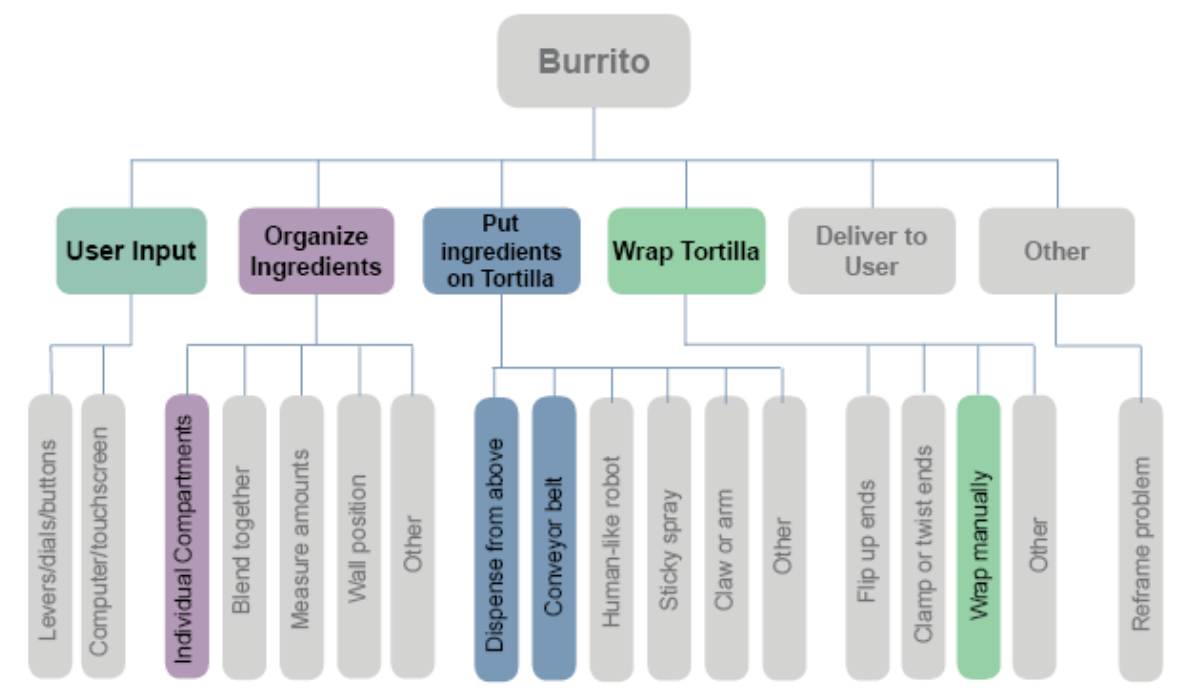

Figure 4: Example of coding the concept featured in Figure 3.

To compare each student's set of Brainstorming ideas and set of Design Heuristics ideas, we counted how many of the 6 main categories were addressed in each. An example of this analysis on Student A's data is shown in Table 3. Concept numbers 1-5 correspond to ideas generated using Brainstorming while concept numbers 6-10 correspond to ideas generated using Design Heuristics. In this case, Brainstorming ideas appeared only in User Input, Organize Ingredients, Put Ingredients on Tortilla, and Wrap Tortilla while Design Heuristics appeared across all 6 categories. For this student, a more diverse set of ideas were produced using Design Heuristics.

Table 3: Student A's 10 concepts coded for the 6 main solution categories. Concepts 1-5 correspond to Brainstorming ideas; concepts 6-10 correspond to Design Heuristics ideas.

\begin{tabular}{lll} 
Main Categories & Concept Numbers & Strategies applied \\
\hline User Input & 9 & Design Heuristics \\
Organize Ingredients & $1,2,3,4,5,6,7,8,9$ & Brainstorming, Design Heuristics \\
Put Ingredients on Tortilla & $1,2,3,4,5,6,7,8$ & Brainstorming, Design Heuristics \\
Wrap Tortilla & $1,3,4,5,10$ & Brainstorming, Design Heuristics \\
Deliver to User & - & - \\
Other & - & -
\end{tabular}

The results of the variety tree analysis across all 18 students is shown in Figure 5. Both Brainstorming and Design Heuristics sessions led to a set of concepts across participants that "covered the space" of the six main process steps defined in the problem. In addition, the methods appeared to foster different categories of concepts. In particular, Brainstorming produced a greater emphasis on concepts for "wrapping tortillas," while Design Heuristics led to more concepts involving "Delivery." This suggests Brainstorming resulted in the concepts fitting the specific goals of producing a burrito ("put ingredients in” and "wrap"), while Design Heuristic concepts 
addressed alternative ways to deliver the product through "bigger picture" or "systems" thinking. Design Heuristics also led to more unique concepts falling into the "Other" category. These differing emphases in functions addressed suggests that conducting idea generation using multiple methods may benefit students by leading them to consider new concepts. Further analysis will be necessary in order to determine how individual students broadened their design trees by using these two different idea generation methods.

\section{Percentage comparison between categories}

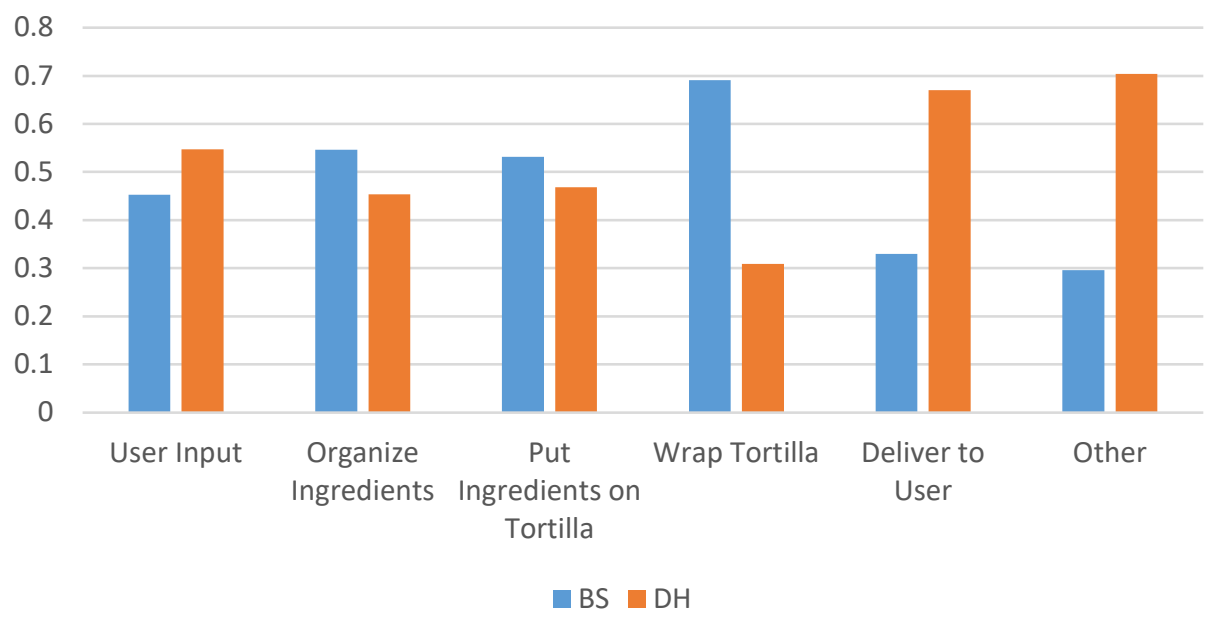

Figure 5: Proportion of variety tree categories covered during Brainstorming vs. Design Heuristics idea generation.

\section{Frequency of Concepts}

To explore another way to identify the difference between Design Heuristics and Brainstorming processes, we categorized each concept as describing either a system or a single component. A system is defined as an idea which addresses two or more of the main categories from variety tree analysis. A component is counted as an idea which addresses only one of those categories. For example, Figure 6 was rated as a system because it addresses 'Organize Ingredients,' 'Wrap Tortilla,' and 'Put Ingredients on Tortilla.' This represents a prototypical example of a concept including all three of these components.

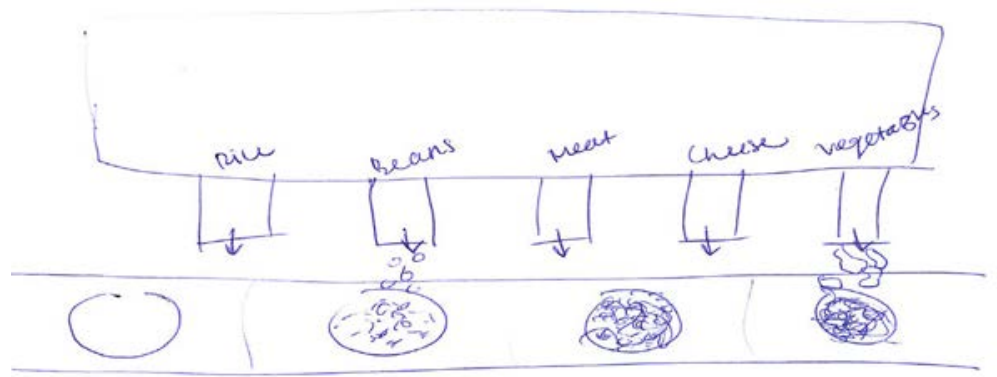

"Conveyor belt w/ tortillas, ingredients of choice are released as tortillas pass beneath."

Figure 6: Example student drawing and description for a device with individual containers for ingredients, ingredients that dispense from above, and a conveyor belt. 
After coding for systems and components in students' concepts, we found that $94 \%$ of students generated this exact concept at some point in their ideation session. Overall, 67\% of students had this concept as their first idea generated. Many iterations of this concept additionally included folding the burrito and delivering it to the user. This specific concept represents $24 \%$ of the 164 concepts in the study. What is it about this idea that causes students to consider it? Likely, knowledge of existing non-automated methods serve as a reference for students attempting a novel design. This suggests analogical reference as a way of conceiving new designs[31].

An additional question to consider is whether this predominant concept influenced the generation of alternative concepts. Based on the notion of "fixation," past studies have found designers often perseverate on concept ideas when attempting to generate novel ones[18, 32]. Our analysis suggests that for 58\% of those who generated this idea during Brainstorming, no variations on it appeared during idea generation with Design Heuristics. This suggests that, despite its frequency in the Brainstorming session, students did not appear to fixate on this familiar concept when using the Design Heuristics.

After coding all concepts for whether they addressed a system or a single component, we found that ideas generated using Design Heuristics fell into component concepts rather than systems concepts, while Brainstorming ideas more frequently describe a system, and shown in Table 4.

Table 4: The number of concepts generated with Brainstorming and Design Heuristics methods, and their focus on to Components compared to Systems.

\begin{tabular}{l|lll} 
& Brainstorming & Design Heuristics & Total \\
\hline $\begin{array}{l}\text { System- } \\
\text { focused }\end{array}$ & 74 & 10 & 110 \\
\hline $\begin{array}{l}\text { Component- } \\
\text { focused }\end{array}$ & 37 & 40 & 50
\end{tabular}

\section{Discussion}

The diversity analyses identified several important qualities of concepts for this design problem. Students grouped their concepts into three or more categories, reflecting their identification of similarities in their designs. Variety Tree analyses uncovered differences in the frequency of solutions including specific processes and functions to achieve them. These two diversity analyses approached the data from different lenses, yet with similar results, with both suggesting more diversity in concepts generated with Design Heuristics. In addition, the emergent distinction between system and single component foci in concepts showed that Brainstorming processes led to more holistic, system-level concepts, while Design Heuristics concepts showed greater emphasis on component design. These findings align with prior research demonstrating that Design Heuristics support consideration of concept elaboration.

In this study, there were many cases where the concepts generated using Design Heuristics specified how a function could be achieved. This suggests that one aspect of learning from Design Heuristics is this focus on a variety of ways to achieve functions. This suggestion has not been identified in past research on Design Heuristics. In comparison, Brainstorming concepts often described a broader idea focused on a system level. Other ideation approaches, such as 
Morphological Analysis[33], guide designers to attend to functional descriptions. If different idea generation methods have unique emphases in inspiring designs, students may be well served by learning multiple methods to assist them in idea generation. In addition, studies comparing idea generation methods must also attend to how outcomes are measured; based on the emphases within methods, some quantitative measures may fail to identify advantages with each method.

Another pattern emergent in the data was a single concept generated by almost all (94\%) of the students. Based on previous studies of fixation[18], [32] this pattern suggests that measuring how students are able to generate further concepts apart from this concept could be a compelling way to measure the impact of idea generation strategies. Because fixation is so prevalent in studies of design,[34] further analysis is needed to understand the impact of ideation methods such as Brainstorming and Design Heuristics in overcoming fixation.

Finally, though the study design did not allow a comparison of the "fluency" or number of concepts generated with the two methods, the protocol supports the notion that combining methods (at least in this format) may help to support the generation of multiple ideas. After a period of Brainstorming, it is likely that less active generation takes place; in studies of idea generations in non-design settings, the exhaustion of ideas after a short period of brainstorming is a prevalent finding[35]. Studies of persistence show people underestimate their ability to generate further ideas, which may cause them to prematurely end their attempts[36]. The success of generating more ideas in the second session while using Design Heuristics may help students to envision idea generation as an unlimited process involving a variety of approaches.

Limitations of this work include the small sample included, and the single design problem used in the study. It is possible that consideration of more concepts, and of other design problems that vary in solutions, may uncover further information on the impact of ideation methods on the diversity of concepts generated. As a naturalistic study within an educational session, comparing alternative methods was moderated by the need to provide the same learning benefit to all participants. As a qualitative study, it is important to employ triangulation with multiple methods of analysis to more fully characterize design outcomes[37]. Further analyses may determine ways of measuring the 'Other' category in these designs. The unique or infrequent concepts in these 0 categories may represent interesting and novel ideas that are valuable outcomes from ideation techniques. It is possible that generating an idea falling in the 'Other' category may indicate an "out-of-the-box," non-obvious idea desirable during concept generation. Generating unique ideas is encouraged in design[11], and further examination of unusual ideas may help us to understand what fosters them. In addition, the order of idea generation method in our study was Brainstorming followed by Design Heuristics. As a result, we cannot directly assess the impact of alternate ordering of techniques. However, in a prior study, Design Heuristics use was compared to Brainstorming by separate groups, and concepts generated using Design Heuristics were more elaborate and practical than during Brainstorming [17]. The present study explores a sequence of methods where openended, natural "brainstorming" is followed by the more structured support of the Design Heuristics tool. Since more ideas logically lead to better candidate concepts, the findings here show that Design Heuristics are effective in supporting continued idea generation even after an initial ideation session. Further investigations are needed to understand the impact of Design Heuristics on design outcomes, with and without the use of complementary methods. 
The implications of this study for engineering education include the finding that different idea generation methods may assist designers in employing different idea generation strategies. For example, Brainstorming may be helpful when first beginning a project because existing solutions and initial ideas can be expressed. Then, Design Heuristics may support idea generation once spontaneous ideas are exhausted, and a more directed exploration of the design space is needed. By examining differing idea generation methods, a greater understanding of how to support novice engineers as they learn to generate ideas may be uncovered.

\section{Conclusion}

We used three analytical lenses to explore differences among novice designers' concepts generated using Brainstorming and Design Heuristics. Understanding the impact of these ideation tools in engineering education is important since Brainstorming is the most widely implemented tool across domains[15], and Design Heuristics has been empirically documented to facilitate idea generation in engineering students.[20]'[27] Our diversity analysis showed initial differences between ideation tools, while further analysis is required to understand how each method supports diversity in the concepts generated. The results suggest that Design Heuristics are more likely to focus the student's attention on the design of a component to serve a function, while Brainstorming tended to focus students on generating holistic systems. The results suggest why different idea generation tools are important for novice engineers, and which in contexts students may find the tools most valuable. This investigation has value for educators who are considering how to foster varied concept development in the early phases of design.

\section{References}

[1] D. P. Crismond and R. S. Adams, “The Informed Design Teaching and Learning Matrix,” J. Eng. Educ., vol. 101, no. 4, pp. 738-797, Oct. 2012.

[2] S. R. Daly, S. Yilmaz, L. . Murphy, and A. Ostrowski, "Tracing problem evolution - factors that impact design problem definition.,” Des. Think. Res. Symp. 11 Peer Rev., Nov. 2016.

[3] J. Kim and D. Wilemon, "Focusing the fuzzy front-end in new product development.," $R D$ Manag., vol. 32, no. 4, pp. 269-279, 2002.

[4] Y.-C. Liu, A. Chakrabarti, and T. Bligh, “Towards an 'ideal' approach for concept generation,” Des. Stud., vol. 24, no. 4, pp. 341-355, Jul. 2003.

[5] E. Z. Opiyo, "An Approach to Represent and Communicate Product or System Design Ideas at the Fuzzy-Front End of the Design Process,” Systems, vol. 4, no. 1, p. 8, Jan. 2016.

[6] J. Malmqvist, P. Young Y., S. Hallstrom, J. Kuttenkeuler, and T. Svensson, "Lessons learned from design-build-test-based project Course,” 32 Proc. Des. 2004 8th Int. Des. Conf. Dubrov. Croat., 2004.

[7] T. Brown, “Design Thinking,” Harv. Bus. Rev., pp. 84-95, 2008.

[8] D. H. Cropley, "The role of creativity as a driver of innovation.," Proc. 2006 IEEE Int. Conf. Manag. Innov. Technol. Singap.

[9] J. Duderstadt, "Engineering for a changing world: A roadmap to the future of engineering practice, research, and education.," Ann Arbor MI Millenn. Proj., 2008.

[10] S. Sheppard, K. Macatangay, A. Colby, and W. Sullivan, Educating engineers: Design for the future of the field. San Francisco: Jossey-Bass, 2009. 
[11] Ö. Akin, “Necessary conditions for design expertise and creativity,” Des. Stud., vol. 11, no. 2, pp. 107-113, Winter 1990.

[12] D. R. Brophy, "Comparing the Attributes, Activities, and Performance of Divergent, Convergent, and Combination Thinkers,” Creat. Res. J., vol. 13, no. 3-4, pp. 439-455, Oct. 2001.

[13] S. Yilmaz, C. Seifert, S. Daly, and R. Gonzalez, "Design Heuristics in innovative products,” J. Mech. Des., vol. 138, no. 7, 2016.

[14] S. Ahmed, K. M. Wallace, and L. T. Blessing, "Understanding the differences between how novice and experienced designers approach design tasks,” Res. Eng. Des., vol. 14, no. 1, pp. 1-11, 2003.

[15] A. F. Osborn, Applied imagination: principles and procedures of creative thinking, 3, revised ed. Charles Scribner's Sons, 1957.

[16] N. Coates, I. Cook, and H. Robinson, "Idea Generation Techniques in an Industrial Market.",” J Mark Pr., vol. 3, no. 2, pp. 107-118, 1996.

[17] S. R. Daly, C. M. Seifert, S. Yilmaz, and R. Gonzalez, "Comparing Ideation Techniques for Beginning Designers,” J. Mech. Des., vol. 138, no. 10, pp. 101108-101108-12, Aug. 2016.

[18] N. Cross, "Design cognition: Results from protocol and other empirical studies of design activity," in Design Knowing and Learning: Cognition in Design Education, C. Eastman, W. Newstatter, and M. McCracken, Eds. Oxford, UK: Elsevier, 2001, pp. 79-103.

[19] A. T. Purcell and J. S. Gero, "Design and other types of fixation,” Des. Stud., vol. 17, no. 4, pp. 363-383, Oct. 1996.

[20] S. R. Daly, S. Yilmaz, J. L. Christian, C. M. Seifert, and R. Gonzalez, "Design Heuristics in Engineering Concept Generation,” J. Eng. Educ., vol. 101, no. 4, pp. 601-629, 2012.

[21] P. B. Paulus and H.-C. Yang, "Idea generation in groups: A basis for creativity in organizations,” Organ. Behav. Hum. Decis. Process., vol. 82, no. 1, pp. 76-87, May 2000.

[22] S. Yilmaz, C. M. Seifert, and R. Gonzalez, "Cognitive heuristics in design: Instructional strategies to increase creativity in idea generation,” Artif. Intell. Eng. Des. Anal. Manuf., vol. 24, no. 03, pp. 335-355, Jul. 2010.

[23] S. Yilmaz, S. R. Daly, C. M. Seifert, and R. Gonzalez, "Evidence-based design heuristics for idea generation,” Des. Stud., vol. 46, pp. 95-124, Sep. 2016.

[24] S. Yilmaz, C. M. Seifert, J. L. Christian, S. R. Daly, and R. Gonzalez, "Design heuristics observed in innovative products.," Iowa State University and University of Michigan., 2012.

[25] C. M. Gray, C. M. Seifert, S. Yilmaz, S. R. Daly, and R. Gonzalez, "What is the content of 'Design Thinking'? Design Heuristics as conceptual repertoire.,” Int. J. Eng. Educ., vol. 32, no. 3B, pp. 1349-1355, 2016.

[26] C. M. Seifert, L. S. Chapman, J. K. Hart, and P. Perez, "Enhancing intrinsic motivation in health promotion and wellness.,” Am. J. Health Promot., vol. 26, no. 3, pp. TAHP1TAHP10.

[27] J. L. Christian, S. R. Daly, S. Yilmaz, C. M. Seifert, and R. Gonzalez, "Design Heuristics to Support Two Modes of Idea Generation: Initiating Ideas and Transitioning Among Concepts,” presented at the Annual Conference of American Society of Engineering Education, San Antonio, Texas, 2012.

[28] S. R. Daly, J. L. Christian, S. Yilmaz, C. M. Seifert, and R. Gonzalez, “Assessing Design Heuristics for Idea Generation in an Introductory Engineering Course,” Int. J. Eng. Educ., vol. 28, no. 2, pp. 1-11, 2012. 
[29] J. M. Kramer, S. R. Daly, S. Yilmaz, C. M. Seifert, and R. Gonzalez, "Investigating the Impacts of Design Heuristics on Idea Initiation and Development," Adv. Eng. Educ., vol. 4, no. 4, pp. 1-26, 2015.

[30] J. J. Shah, N. Vargas-Hernandez, and S. M. Smith, "Metrics for measuring ideation effectiveness," Des. Stud., vol. 24, no. 2, pp. 111-134, Mar. 2003.

[31] J. L. Kolodner, R. L. Simpson, and K. Sycara-Cyranski, "A process model of cased-based reasoning in problem solving.," Sch. Inf. Comput. Sci. Ga. Inst. Technol., p. 6.

[32] D. G. Jansson and S. M. Smith, “Design fixation,” Des. Stud., vol. 12, no. 1, pp. 3-11, 1991.

[33] N. Cross, Engineering Design Methods: Strategies for Product Design, 4th ed. West Sussex, England: Wiley, 2008.

[34] N. Crilly, "Fixation and creativity in concept development: The attitudes and practices of expert designers," Univ. Camb. Dep. Eng. Trumptington Str. Camb. CB2 IPZ UK.

[35] E. P. Torrance, "Encouraging Creativity in the Classroom," Duboque Iowa William C Brown Publ., 1970.

[36] B. J. Lucas and L. F. Nordgren, "People underestimate the value of persistence for creative performance.,” J. Pers. Soc. Psychol., vol. 109, no. 2, pp. 232-243, 2015.

[37] N. L. Leech and A. J. Onwuegbuzie, "An array of qualitative data analysis tools: A call for data analysis triangulation,” Sch. Psychol. Q., vol. 22, no. 4, pp. 57-584, Dec. 2007. 